\title{
The Application of Microwave Technology in Chemistry and Chemical Engineering
}

\author{
Yunshen Zhang \\ Department of Petrochemical Engineering, Puyang Vocational and Technical College, puyang \\ Henan, P. R. China 457000
}

Key words: microwave technology; chemical industry; application

\begin{abstract}
Microwave can promote the chemical reaction, which has great influence on the chemical reaction process. It has been widely concerned by its unique advantages. Microwave technology can be used in organic synthesis, material synthesis, sample analysis, waste water treatment and other fields, the technology heating speed, high efficiency, and its low cost and good environmental benefits, is widely used in chemical and chemical industry in recent years. In this paper, the application of microwave technology in chemical industry was discussed, and practical application strategy was put forward, which could provide reference for the application of microwave technology in chemical industry.
\end{abstract}

\section{Introduction}

Microwave is a kind of electromagnetic wave, usually the wavelength between 1-1000 mm, the frequency is between $0.3-300 \mathrm{GHz}$. In order to avoid microwave interference in different fields, the international conventions on the microwave frequency is strictly regulated for industrial, civil, scientific research in the frequency of heating and drying are different, $2450 \mathrm{MHz}$ is equivalent to $12.2 \mathrm{~cm}$ microwave wavelength, which is also the most widely used frequency. At the end of the 19th century, Hertz proved the existence of electromagnetic waves, after 1936, American scientists confirmed that electromagnetic waves can be hollow metal tube in the transmission, so the rapid development of microwave technology has also been widely used in many fields. Therefore, the microwave technology in the chemical and chemical applications, the chemical and chemical industry's sustainable development has great practical significance.

\section{The development of microwave technology}

Since the Second World War in September 1939, the rapid development of radar technology, people have also been a lot of knowledge of microwave radiation, microwave radiation has also been rapid expansion of use. In the end of 1960s, microwave irradiation technology is widely used in various fields, such as sterilization, preparation of analytical samples, waste treatment, protein and peptide bond hydrolysis, etc., in chemical and chemical applications began in the temperature jump experiment, the experiment applied microwave heating, 1970s there have been reports on the microwave application of chemical research.

In 1986, some scholars found that the traditional microwave oven chemical reaction can be accelerated quickly, chemical synthesis reaction can be achieved in a short time the desired effect. People also began to focus on microwave heating to achieve chemical reactions, but also a lot of research on this technology, has been developed into microwave chemistry. In 1992, the first World Congress of Microwave Chemistry was held in the Netherlands. This was the first time that microwave chemistry was born. After that, many scientists carried out a wide range of research, including inorganic chemistry, organic chemistry, analytical chemistry, polymer chemistry, catalysis chemical, etc., especially in organic synthesis of microwave technology applications. 


\section{Microwave technology applications}

\subsection{Microwave technology in organic synthesis applications}

Early microwave radiation is used in organic synthesis reaction, the last 60 years of electromagnetic pulse to the acrylic acid, acrylic acid, methacrylic acid polymerization. (1) usually microwave-assisted organic reaction mechanism is divided into two kinds, one for the thermal effect (1), the microwave-assisted organic synthesis reaction mechanism, the microwave-assisted organic synthesis reaction mechanism, : Microwave heating speed and uniform, showing no temperature gradient, no hysteresis effect. The microwave may cause the instantaneous hyperthermization of the solvent, and the liquid may reach a higher temperature before boiling. Microwave can effectively accelerate the chemical reaction, which can be polar organic heating; the other for the non-heating effect: the microwave will accelerate the molecular motion of the reactants, the reactant temperature will rise rapidly, during which ions and polar molecules Lorentz force to promote the special movement between particles, resulting in non-heating effect. (2) microwave organic synthesis reaction technology: This technology under the different organic reactions, microwave response to specific equipment and reaction technology to achieve, usually microwave organic synthesis reaction technology for the microwave closed synthesis technology, in 1986 some scholars use microwave technology Organic synthesis of the way is closed synthesis technology. Microwave closure synthesis reaction technology emphasizes the reactant sealing reaction device placed in the microwave source, when the reaction is completed, the reactor cooling product purification, the technology is a high temperature, high pressure reaction of a technology. Microwave heating solution in the device can be refluxed, PTFE tube with the reactor connected to each other, and then the pipeline to provide an inert gas for the reaction, then can effectively protect the reaction system.

\subsection{Microwave technology in the synthesis of materials}

There are many microwave methods for synthesizing chemical materials, such as microwave homogeneous precipitation method and microwave coprecipitation method, microwave hydrothermal method and microwave sintering method. Often olivine-type structure of lithium iron phosphate lithium-ion battery cathode material, which has a high theoretical capacity, the voltage platform is also reasonable and reasonable, good cycle performance, to achieve low prices, material safety and environmental protection. Microwave synthesis of lithium-ion battery cathode material, can effectively shorten the synthesis time, low energy consumption, cadmium sulfide for semiconductor materials, is widely used in solar energy conversion and non-linear optics, photoelectron chemical batteries and other materials synthesis.

\subsection{Microwave technology in the sample analysis of the application}

The microwave technique in the sample analysis can be divided into three aspects: microwave drying, microwave digestion and microwave extraction: (1) Microwave drying is the internal heating mode, that is, microwave heating in microwave drying, Water molecules will change with the alternating high-frequency electric field to achieve interactive changes, then there will be violent collision and friction, to promote the local microwave energy into molecular kinetic energy, the form of heat to the performance of water temperature also shows that the material will Leave, then you can achieve the purpose of drying. After the microwave is dried and absorbed, the internal energy of the dielectric material is converted to heat. Microwave drying is the electromagnetic wave as the heating source, and the need to dry the material is the heating element. Microwave drying speed, the short time required, high thermal stability, uniform heating, high product quality, sterilization effect of a good insecticide. In order to make full use of microwave drying and the characteristics of the existing drying technology, microwave hot air drying machine microwave vacuum drying has been developed and widely used. (2) Microwave digestion is a mixture of acid and sample polarity of the molecules in the microwave under the electromagnetic field will change the polarity, then it will have a microwave digestion and microwave digestion is the use of 
microwave, Bond vibration and tear, the particles will be friction and collision between each other, then produce a lot of heat, making the acid and the sample fully reacted. The contact with the heated sample of water and acid will produce more heat than the sample surface, then there is a strong thermal convection to achieve mixing and removal of sample particles have been dissolved surface of the inactive surface layer, the sample And the acid contact surface will be quickly updated to achieve the purpose of sample dissolution acceleration. Microwave digestion has been used in many fields, such as biotechnology, cosmetics, environmental protection, materials synthesis. Some scholars to microwave microwave digestion milk samples, while optimizing the microwave digestion conditions, the test available: microwave digestion of milk powder in the relative standard deviation of copper, spike recovery, this approach is simple and convenient, applicability, usually in this way the analysis of milk And the amount of copper contained in dairy products; (3) Microwave extraction is the use of microwave heating can be used to extract the microwave energy, which can quickly improve the extraction efficiency. There are different substances in the microwave field, the dielectric constant is different, the degree of absorption of microwave energy is also different, the resulting heat there is a certain difference, the heat can be transmitted is different, these differences in the extraction system The local composition and the matrix material appear area uneven heating, and this non-uniformity will cause the extract in the matrix separation. Microwave extraction has been used in environmental samples, soil, plants and animals in the extraction of active ingredients, such as microwave extraction can be within 20 seconds in the days of tincture extraction of flavonoids, the total extraction up to $1.883 \%$; or in marine sediments in the half 12 hours within the sample processing, while the traditional technology is only 16 hours to deal with one, showing that the microwave extraction technology is fast, and its extraction efficiency, the resulting product quality, low cost. Microwave technology has been used for derivatization, regeneration, analysis, sample online processing.

\subsection{Microwave technology in waste water treatment applications}

Microwave heating does not require heat transfer, or internal and external heat, heat transfer will not be heat loss, this advantage can be used to deal with microwave technology, sludge or organic pollutants. Application of microwave technology in waste water treatment is: (1) Microwave-induced oxidation, the oxidation reaction is a strong absorption of microwave sensitizers to microwave energy is not directly absorbed in the microwave absorption of organic compounds, and thus the catalytic reaction, The technical realization is in the microwave induced oxidation process preparation of the appropriate catalyst. Some scholars use ferric oxide as a catalyst to treat waste water, the results show that about 50\% COD and $80 \%$ of the color water can be removed; some scholars to self-made Fe- $\mathrm{Zr}$ as a catalyst, and $\mathrm{H} 2 \mathrm{O} 2$ as an oxidant treatment of n-butyric acid (2) Microwave-induced UV treatment of waste water, some scholars to sodium hypochlorite as oxidant, and then to the waste water treatment, the use of micro-wave technology can be effective in the treatment of waste water, the TOC removal rate of $95 \%$ The results show that the effect of waste water treatment is better if the dosage of sodium hypochlorite is increased, but the dosage of sodium hypochlorite is strictly controlled; (3) The microwave irradiation (microwave irradiation) Regeneration, in this way is to achieve the adsorption of activated carbon or adsorbent pollutants, and then saturated adsorbent in the microwave field deep radiation, the pollutants fully degraded, microwave radiation regeneration waste water treatment costs less, and the adsorbent is renewable ; (4) Direct microwave radiation is directly to the microwave radiation containing pollutants adsorbate, the water pollutants fully removed, this way with chemical oxidation, photocatalysis and other applications in order to effectively improve the pollutant removal effect.

\section{The development prospects of microwave technology}

Microwave technology is widely used in chemical and chemical industry, the microwave heating in chemical synthesis is very superior, and its heating speed, can improve the reaction rate; heat source and reactants, solvents can not direct contact, the reaction parameters can be effectively controlled, 
Safety is also controlled; this technique can be selectively heated to selectively heat the microwaves if the compounds present in the reaction mixture have different absorption microwave capabilities to ensure that the yield is increased and the incidence of side reactions is also reduced ; The level of automation with the rapid development of science and technology to enhance productivity is also rising. Microwave technology is fast and efficient, low energy consumption, and little pollution to the environment, for the green synthesis method, can stimulate the high energy, background radiation than the traditional low, can be widely used in various fields. However, there are some limitations, such as the high cost of the microwave reactor, the shorter depth of the microwave radiation through the liquid medium, the large size of the reactor, and the difficulty of the experiment. In the modern society, the plant microwave system is the largest For the $100 \mathrm{~L}$, these problems are the future development of microwave technology need to attach great importance to the problem.

\section{Concluding remarks}

With the rapid development of modern society and economy, microwave technology also will be rapid development, the technology heating speed, high efficiency, the cost is not much, the impact on the natural environment is small, the technology has been used for navigation , Communications, military, weapons and other fields. However, microwave technology is still emerging science and technology, China's microwave technology is still the initial stage of development, its application is still a lot of shortcomings, so the microwave technology in the chemical and chemical applications on the chemical and chemical industry's sustainable development has great practical significance . In this paper, the development of microwave technology, the application of microwave technology, the development prospects of microwave technology, as a microwave technology in the chemical and chemical industry to provide a reference basis.

\section{References}

[1] Wang Chao. Development and application of microwave technology [J]. Silicon Valley, 2010 (06).

[2] Tong Xing, Xiao Xiaohua, Deng Jianchao, Wang Jiayue, Li Gongke.Application of Low Temperature Microwave Technology in Chemical Research [J].

[3] Liu Yuting, Zhou Ying, Yin Dawei, banquet new.Application of microwave technology in chemical industry [J]. Chemistry World, 2010 (08).

[4] Zhou Weilei, Bai Suozhu, Wang Rui.Application of microwave technology in chemical field [J]. Guangzhou Chemical Industry, 2015 (10).

[5] Cheng Li, Cai Jing, Yin Hongmei, etc.Application of Microwave Chemical Reactor in Pharmaceutical Chemistry [J] .Journal of Experimental Science and Technology, 2011 (03).

[6] Lv Weizhong, Liu Bo, Wang Fang, etc.Synthesis and Characterization of 2-Phenylbenzimidazole under Microwave Irradiation [J]. Fine Chemical Industry, 2009 (26).

[7] Xu Hui, Liu Weiping, Su Yuanzhi, et al. Microwave synthesis and properties of LiFePO4 / C cathode materials [J]. Materials Review B, 2011 (25).

[8] Cheng Li, Cai Jing, Yin Hongmei, Li Feng, He Guangli, Wang Hong, Deng Ruwei, Tu Li, Wu Yong, Yang Zuxing, Tang Jie Application of Microwave Chemical Reactor in Medicinal Chemistry [J]. (03).

[9] Cao Yingying, Li Jianxi, Zheng Zhenzhong, Zhang Ping.Simulation of microwave heating water [J]. Industrial Heating, 2011 (02).

[10] Zheng Zhen, Zheng Jian, Zheng Zhenhong.Discussion on industrial microwave heating and energy saving and emission reduction [J]. China Hi-Tech Enterprise, 2011 (34). 